\title{
Dual effects of heat stress on tumor necrosis factor- $\alpha$-induced hepatocyte apoptosis in mice
}

\author{
Motohiro Imao, Masahito Nagaki and Hisataka Moriwaki \\ Department of Gastroenterology, Gifu University Graduate School of Medicine, Gifu, Japan
}

\begin{abstract}
The major heat shock protein, HSP70, plays a critical role in cell survival in response to stress, possibly by inhibiting a number of antisurvival pathways. However, heat stress (HS) and HSPs also sensitize cells to certain apoptotic stimuli, such as TNF- $\alpha$. To clarify the relations between HS and apoptosis, we examined the differential effects of the intensity of HS on liver injury and apoptosis induced by TNF- $\alpha$ in mice. TNF- $\alpha$ was injected into D-galactosamine (GaIN)-sensitized mice that were pretreated with or without HS. Liver injury was assessed biochemically and histologically. In GalN-sensitized mice, application of HS for 7 days led to significant enhancement of TNF- $\alpha$-induced hepatotoxicity, despite upregulation of HSP70 in the liver. In contrast, application of HS for 1 day led to attenuation of TNF- $\alpha$-induced liver injury. Repeated HS decreased the levels of the FLICE inhibitory protein short (FLIPS) and activated caspase-8 in the liver. The caspase-8 inhibitor Z-IETD-FMK effectively protected both the nontreated and HS-pretreated mice from the hepatotoxicity induced by GaIN/TNF- $\alpha$. HS shows dual effects on TNF- $\alpha$-induced hepatocyte apoptosis. Exposure to repeated HS, but not to single HS, leads to enhancement of TNF- $\alpha$-induced hepatocyte apoptosis via the interaction of FLIP and caspase-8.
\end{abstract}

Laboratory Investigation (2006) 86, 959-967. doi:10.1038/labinvest.3700451; published online 10 July 2006

Keywords: heat shock protein; caspase-8; FLICE inhibitory protein

Exposure of cells to any of a wide variety of stressors, such as heat stress (HS), hypoxia, hydrogen peroxide, inflammation, and ischemia, results in similar accumulation of the stress proteins known as heat shock proteins (HSPs). ${ }^{1,2}$ The main function of HSPs is to operate as intracellular chaperones for aberrantly folded or mutated proteins and to provide cytoprotection against the stress conditions. The HSP70 protein family is the abundant group in eukaryotic cells, and contains both constitutive and inducible forms that are localized to different intracellular organelles. ${ }^{1}$ The heat-inducible form of HSP70 is expressed at low basal levels but is rapidly induced by heat or other stresses. HSP70 can be induced in liver, cardiac muscle, renal cells, skeletal muscle, and brain tissue. A number of studies have indicated that HSP70 protects cells and tissues from injury under various pathological conditions via inhibition of procaspase-9 to the Apaf-1/apoptosome complex, ${ }^{3,4}$ protection of mitochondrial membranes, ${ }^{5}$ inhibition of the activation of c-Jun N-terminal kinase (JNK), ${ }^{6-8}$ or inhibition of

Correspondence: $\mathrm{Dr} \mathrm{M}$ Nagaki, MD, PhD, Department of Gastroenterology, Gifu University Graduate School of Medicine, 1-1 Yanagido, Gifu 501-1194, Japan.

E-mail: mnagaki@cc.gifu-u.ac.jp

Received 22 January 2006; revised 1 June 2006; accepted 2 June 2006; published online 10 July 2006
BID cleavage. ${ }^{9}$ In addition to these in vitro studies, there are several reports clearly demonstrating that HSP70 protects cells in vivo. HS has been shown to prevent lethality induced by lipopolysaccharide (LPS) or tumor necrosis factor- $\alpha$ (TNF- $\alpha$ ) in rats and mice ${ }^{10-12}$ This protection has also been correlated with the upregulation of HSP70 in several organs. ${ }^{10}$

TNF- $\alpha$ and the Fas ligand are cell-death mediators that act by binding to their responsive receptors, two TNF receptors (TNFR) TNFR1 and TNFR2, and Fas, respectively, and induce apoptosis in a variety of cell types. Both TNFR1 and Fas share a related Cterminal intracellular domain referred to as the 'death domain', and use similar types of associated molecules in the signal transduction pathway leading to apoptosis. ${ }^{13}$ Fas recruits the Fas-associated death domain protein (FADD), and TNFR-1 recruits the TNFR-associated death domain protein (TRADD), which in turn binds FADD. FADD is then associated with procaspase-8, resulting in its activation. Caspase-8 then functions as an apical caspase, leading to the processing of effector caspases such as caspase-3, -6, and -7 (extrinsic pathway). The other caspase cascade, which is known to be activated by genotoxic stressors such as UV irradiation and anticancer drugs, is triggered by the release of cytochrome $c$, which binds to both the cytoplasmic scaffold, known as Apaf-1, and to procaspase-9. The 
binding to procaspase-9 leads to activation of caspase-9, which in turn activates downstream caspase-3, -6, and -7 (intrinsic pathway).

Even though it has been well established that HSPs inhibit various types of apoptosis, some studies have demonstrated that HS or elevated HSP70 potentiates cell death following various stimuli. Elevated HSP70 or overexpression of heat shock transcription factor 1 enhances Fas-mediated cell apoptosis. ${ }^{14,15}$ HS also sensitizes AML cells and endothelial cells to apoptosis. ${ }^{16,17}$ However, the precise mechanism by which HS promotes cell death rather than protects cells from apoptosis remains unclear. Recently, Tran et $a{ }^{18}$ have demonstrated that HS sensitizes cells to Fas-mediated apoptosis through downregulation of the FLICE inhibitory protein (FLIP), which is a cytoplasmic protein that has sequence homology with caspase-8 and is capable of inhibiting apoptosis by blocking the recruitment and processing of caspase-8. In further support of the idea that HSP70 promotes apoptosis, it has been reported that HSP70 enhances TNF-mediated apoptosis by binding I $\kappa \mathrm{B}$ kinase $\gamma$ and impairing NF- $\kappa \mathrm{B}$ survival signaling. ${ }^{19}$

In the present study, to clarify the relations between HS and apoptosis, we examined the effect of different levels of HS exposure on liver injury and apoptosis induced by TNF- $\alpha$ in mice. We demonstrate that application of HS for 7 days in mice induces HSP70 in the liver and leads to significant enhancement of TNF- $\alpha$-induced hepatotoxicity, but application of HS for 1 day leads to attenuation of TNF- $\alpha$-induced liver injury. We further show that the repeated exposure to HS is able to rapidly downregulate the levels of FLIP $_{S}$ and activate caspase-8, and thereby enhance TNFR-mediated apoptosis.

\section{Materials and methods}

\section{Mice}

Pathogen-free, male BALB/c mice (age, 7-8 weeks; weight, 20-25g) were purchased from Japan SLC (Shizuoka, Japan) and maintained for at least 1 week under a 12-h day/night cycle with free access to food and water until the day of the experiment.

\section{Reagents}

D-Galactosamine (GalN) hydrochloride was obtained from Nacalai Tesque (Tokyo, Japan). Recombinant mouse TNF- $\alpha$ was purchased from Genzyme (Cambridge, MA, USA). The fluorogenic substrate Ac-IETD-AMC (Ac-IETD-7-amino-4-methylcoumarin) was obtained from BIOMOL (Philadelphia, PA, USA), and Ac-DEVD-AMC (Ac-DEVD-7-amino-4trifluoro-methylcoumarin) was obtained from Alexis (San Diego, CA, USA). The caspase-8 inhibitor Z-IETD-FMK was obtained from Genzyme. Antibodies for Western blotting were obtained as follows: procaspase-8 and the cleaved product of procaspase8 (p20), FLIP short/long (FLIP S/L $_{\text {L }}$ ), cytochrome c, and actin were from Santa Cruz Biotechnology (Santa Cruz, CA, USA), Akt (Ser-473) and Phospho-Akt (Ser473) were from Cell Signaling Technology (Beverly, MA, USA), HSP70 was from Stressgen Biotechnologies Co. (Victoria, Canada), and anti-rabbit IgG HRP-coupled secondary antibody was from Amersham-Pharmacia Biotech (Buckinghamshire, UK).

\section{Experimental Treatment}

The experiments were conducted in accordance with the institutional guidelines of the Gifu University School of Medicine. Mice were placed in a specially designed cage that enabled roughly twothirds of mouse body surface area to be immersed in a hot water bath controlled to $42.0 \pm 0.5^{\circ} \mathrm{C}$. They received HS for 15 min once a day for 1 day, 3 days or 7 days. GalN was dissolved in sterile, pyrogenfree saline and was administered to mice intravenously (16 mg/mouse). Recombinant mouse TNF- $\alpha$ was diluted with pyrogen-free saline to the required concentrations and intravenously injected into mice $(0.5 \mu \mathrm{g} / \mathrm{mouse})$. Some mice were intraperitoneally treated with $900 \mu \mathrm{g} /$ mouse of the caspase-8 inhibitior Z-IEHD-FMK $30 \mathrm{~min}$ after GalN/TNF- $\alpha$ administration. Control mice received $2 \%$ DMSO in saline at the same time. The mice were anesthetized with ether and killed by drawing blood from the inferior vena cava with heparin-coated syringes. The liver was immediately removed and washed with icecold phosphate-buffered saline (PBS). A part of the dissected liver tissue was frozen in liquid nitrogen for measurements of caspase activity, and for Western blot analysis.

\section{Transaminase Activity}

Serum alanine transaminase (ALT) and aspartate transaminase (AST) were measured with a standard clinical automatic analyzer.

\section{Caspase Activity}

Frozen liver tissues were homogenized in lysis buffer (10 mM HEPES pH 7.4, 2 mM EDTA, 0.1\% 3[(3-cholamidopropyl)dimethylammonio]-1-propanesulfonate (CHAPS), $5 \mathrm{mM}$ dithiothreitol, $1 \mathrm{mM}$ PMSF, $10 \mu \mathrm{g} / \mathrm{ml}$ pepstatin A, $10 \mu \mathrm{g} / \mathrm{ml}$ aprotinin, and $20 \mu \mathrm{g} / \mathrm{ml}$ leupeptin). The homogenates were incubated on ice for $1 \mathrm{~h}$ and centrifuged at $14000 \times g$ at $4{ }^{\circ} \mathrm{C}$ for $20 \mathrm{~min}$, and then the supernanants were collected. Seventy-five $\mu \mathrm{l}$ aliquots of the extracts $(200 \mu \mathrm{g}$ protein) were incubated with fluorogenic peptide substrate Ac-IETD-AMC or Ac-DEVD-AMC at a final concentration of $50 \mu \mathrm{M}$ in $20 \mu \mathrm{l} \times 5$ reaction buffer (50 mM PIPES pH 7.4, $10 \mathrm{mM}$ EDTA, 0.5\% CHAPS, $25 \mathrm{mM}$ dithiothreitol) for $2 \mathrm{~h}$ at $37^{\circ} \mathrm{C}$. The 
change in the fluorescence of released AMC was monitored at 1-h intervals using a Fluoroskan Ascent FL fluorometer (Thermobioanalysis Co., Helsinki, Finland) using an excitation wavelength of $355 \mathrm{~nm}$ and emission wavelength of $460 \mathrm{~nm}$.

\section{Western Blot Analysis}

For Western blot analysis of caspase-8, HSP70, FLIP $_{S}$, FLIP $_{\mathrm{L}}$, Akt, phosphorylated Akt, cytochrome $c$, and actin, frozen liver tissues from mice killed at the indicated time points were homogenized in lysis buffer. Equal amounts of the liver lysates, quantified using a commercially available Bio-Rad protein assay kit (Richmond, CA, USA), were loaded onto each lane of a $12.5 \%$ SDS-PAGE gel. Nitrocellulose membranes (Bio-Rad) were used to transfer proteins and were blocked with $5 \%$ bovine serum albumin buffer overnight. The membranes were probed with Abs against procaspase-8, HSP70, FLIP short/long $\left(\right.$ FLIP $\left._{\mathrm{S} / \mathrm{L}}\right)$, Akt, phosphorylated Akt, cytochrome $c$, and actin, and then incubated with the anti-rabbit or mouse IgG HRP-coupled secondary antibody. Detection was performed with an ECL system (Amersham-Pharmacia Biotech, Buckinghamshire, UK). Analysis of the processed form of caspase-8 (p20) band was performed on a Macintosh computer using the public domain NIH Image program (developed at the US National Institues of Health and available over the Internet from an anonymous FTP at zippy.nimh.nih.gov. or on floppy disk from the National Technical Information Service, Springfield, Virginia, Part No. PB95500195 GI).

\section{Preparation of Cytosolic and Membrane Fractions}

Livers were washed in PBS and disintegrated by seven strokes with a Dounce-type homogenizer in Abuffer (five times volume against liver) $(220 \mathrm{nmol} / \mathrm{l}$ mannitol, $70 \mathrm{mmol} / \mathrm{l}$ sucrose, $50 \mathrm{mmol} / \mathrm{l}$ HEPES $\mathrm{pH}$ 7.0, $5 \mathrm{mmol} / \mathrm{l}$ EGTA, $2 \mathrm{mmol} / \mathrm{l} \mathrm{MgCl} 2$, and protease inhibitors, $0.3 \mathrm{mmol} / \mathrm{l} \mathrm{PMSF}$, and $30 \mu \mathrm{g} / \mathrm{ml} \mathrm{E64).}$
Debris was pelleted by centrifugation at $1000 \times g$ for $5 \mathrm{~min}$. The protein concentration of the resulting postnuclear fraction (supernanant) was adjusted to $8 \mathrm{mg} / \mathrm{ml}$ and then samples were further centrifuged at $100000 \times g$ for $20 \mathrm{~min}$. The resulting supernanant was used as the cytosolic fraction and the pellet as the membrane fraction. The membrane fraction was resuspended and homogenized in the original volume of A-buffer.

\section{Terminal Deoxynucleotidyl Transferase-Mediated dUTP Nick End Labeling Assay}

The liver was excised and immediately fixed with $10 \%$ buffered formalin. A terminal deoxynucleotidyl transferase-mediated dUTP nick end labeling (TUNEL) assay, which relies on incorporation of labeled dUTP at the sites of DNA breaks, was also carried out using $5 \mu \mathrm{m}$-thick sections of the mouse livers. All reagents and buffers for the TUNEL assay were included in an Apop Tag kit (Intergen, Purchase, NY, USA). The procedures were performed according to the manufacturer's instructions. TUNEL-positive hepatocytes are given as a percentage relative to the total number of hepatocytes in each visual field.

\section{Data Analysis}

All values in the figures and text are expressed as the mean \pm s.e. The significance of differences among the mean values was evaluated according to the Mann-Whitney $U$-test.

\section{Results}

\section{Dual Effects of HS on Liver Injury and Hepatocyte Apoptosis Induced by GalN/TNF- $\alpha$}

Intravenous injections of $16 \mathrm{mg}$ of GalN and $0.5 \mu \mathrm{g}$ of $\mathrm{TNF}-\alpha$ into BALB/c mice caused an increase in plasma aminotransferase activity after $6 \mathrm{~h}$ (Table 1).

Table 1 Effects of HS on TNF- $\alpha$-induced liver injury in mice

\begin{tabular}{lcccc}
\hline Pretreatment & $T N F-\alpha(\mu g /$ Mouse $)$ & GalN $(\mathrm{mg} / \mathrm{mouse})$ & ALT (IU/L) & AST (IU/L) \\
\hline- & - & - & $43.3 \pm 1.3$ & $86.6 \pm 3.3$ \\
- & - & 20 & $46.6 \pm 8.8$ & $120.0 \pm 14.1$ \\
- & 0.5 & - & $130.0 \pm 36.5$ & $176.6 \pm 19.9$ \\
HS (1) & - & - & 55.0 & 105.0 \\
HS (7) & - & 20 & $45 \pm 8.6$ & $80 \pm 7.0$ \\
HS (7) & 0.5 & - & $56.6 \pm 3.3$ & $126.6 \pm 12.0$ \\
HS (7) & 0.5 & 20 & $83.3 \pm 12.0$ & $106.6 \pm 16.6$ \\
HS (1) & 0.5 & 20 & $387.7 \pm 1441.8$ & $2054.5 \pm 314.0$ \\
HS (7) & 0.5 & $20775.8 \pm 303^{*}$ & $435 \pm 61.3^{*}$ \\
\end{tabular}

Mice were subjected to HS $\left(42.0+0.5^{\circ} \mathrm{C}\right.$ for $\left.15 \mathrm{~min}\right)$ once a day for 1 day (HS (1)) or 7 days (HS (7)) and injected i.v. with TNF- $\alpha$ and/or GalN $12 \mathrm{~h}$ after the last HS exposure. Blood was collected and serum ALT and AST activities were determined as indices of hepatotoxicity $6 \mathrm{~h}$ after the administration of TNF- $\alpha$ and/or GalN. Data are expressed as the mean \pm s.e. for three to 11 mice.

${ }^{*} P<0.05$ compared to mice treated with GalN/TNF- $\alpha$. 
In mice that were pretreated with HS for 7 days, plasma ALT and AST activities were significantly increased $(P<0.05)$. On the other hand, pretreatment with HS for 1 day significantly reduced aminotransferase activities after GalN/TNF- $\alpha$ injection $(P<0.05)$. We quantified hepatocyte apoptosis by counting cells positive for TUNEL (Figure 1). After administration of GalN and TNF- $\alpha$, a marked increase in the number of apoptotic cells was observed in the livers of mice pretreated seven times with HS as compared to those without HS. However, single treatment with HS significantly reduced the number of TUNEL-positive hepatocytes. These results indicated that the single and repeated HS provided differential effects on liver injury and hepatocyte apoptosis induced by GalN/ TNF- $\alpha$.

Next, to determine whether HS influences the susceptibility of mice to TNF- $\alpha$, we examined the effects of HS for 7 days on the ALT activities of mice
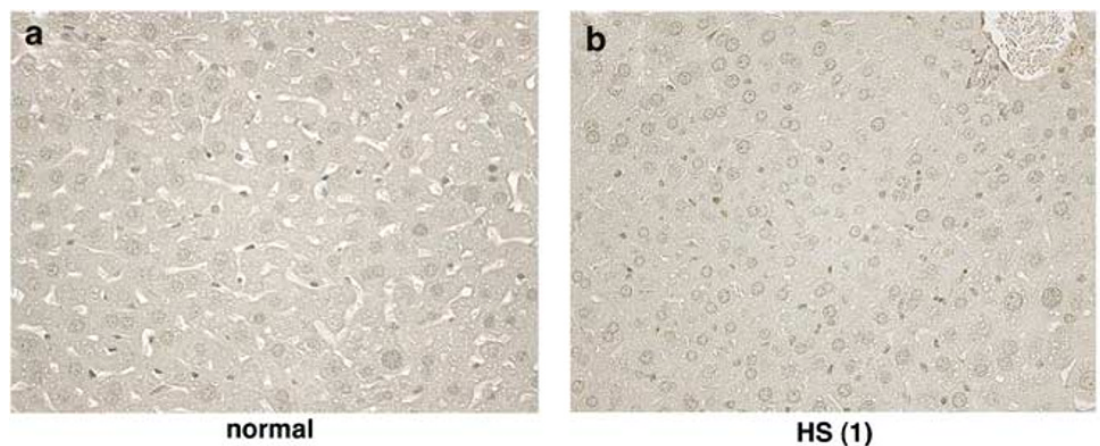

HS (1)

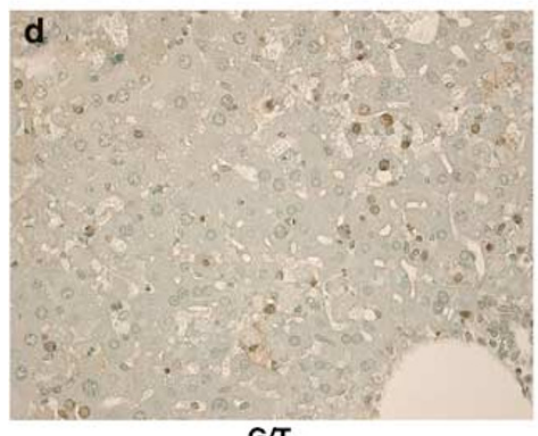

$\mathrm{G} / \mathrm{T}$

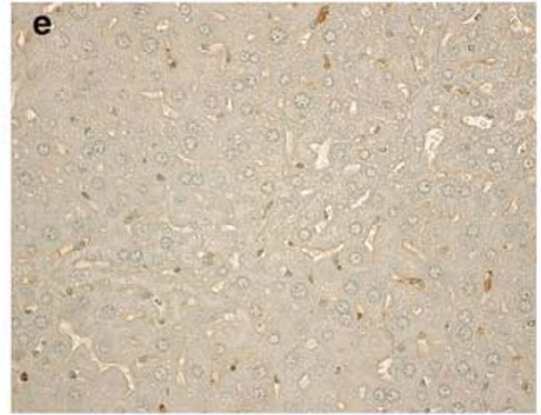

HS (1)+G/T

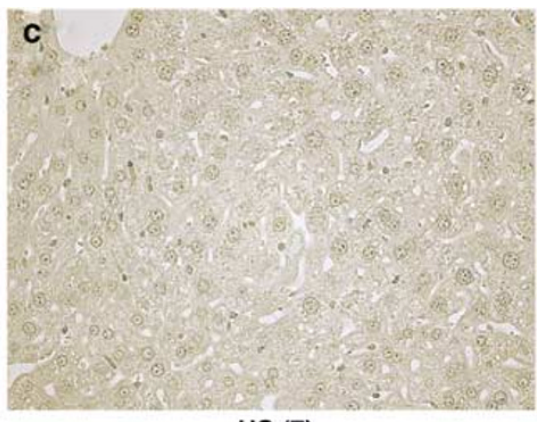

HS (7)

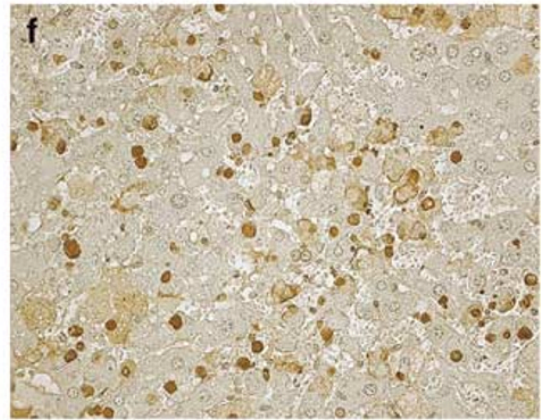

HS (7)+G/T

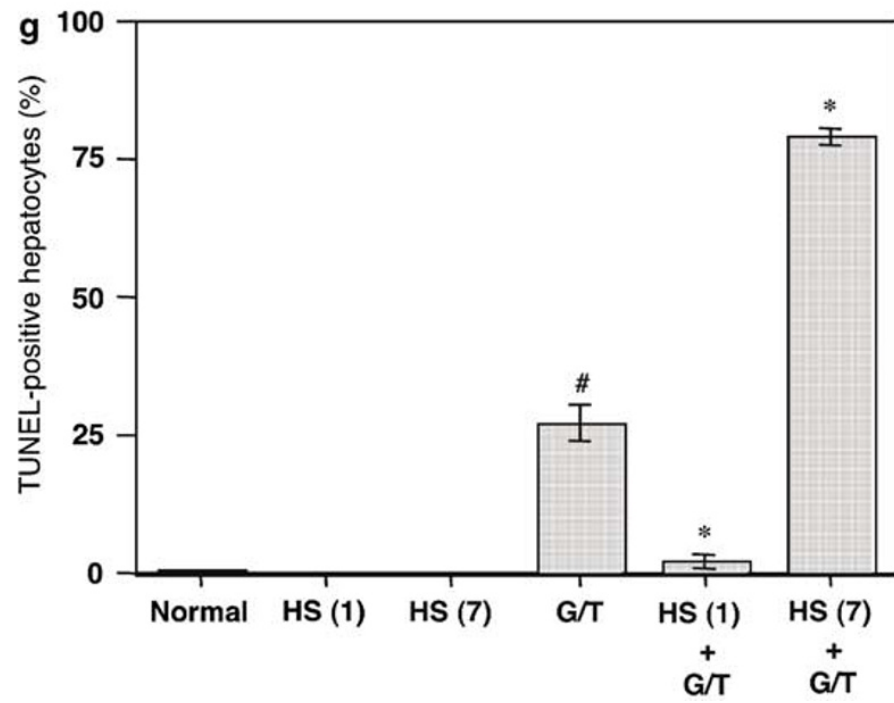

Figure 1 Induction of apoptosis by $16 \mathrm{mg}$ of GalN/0.5 $\mu \mathrm{g}$ of TNF- $\alpha$ in mouse liver pretreated with HS. Mice were subjected to HS $\left(42.0 \pm 0.5^{\circ} \mathrm{C}\right.$ for $\left.15 \mathrm{~min}\right)$ once a day for 1 day (b) or 7 days (c). Control mice were nontreated (a). Mice were injected with GalN/TNF- $\alpha 12 \mathrm{~h}$ after prior HS for 1 day (e) or for 7 days (f) or after receiving no HS (d). The mice livers underwent an in situ terminal deoxynucleotidyl transferase-mediated dUTP nick end labeling (TUNEL) assay to detect apoptotic cells (original magnification $\times 800$ ). (g) Percentages of TUNEL-positive cells among total hepatocytes were determined. Data are expressed as the mean \pm s.e. for $4-14$ mice. ${ }^{*} P<0.05$ compared to mice treated with GalN/TNF- $\alpha$; ${ }^{\#} P<0.05$ compared to normal mice. 
given TNF- $\alpha$ alone (Table 1). However, unlike GalN, HS did not induce liver injury in TNF- $\alpha$-treated mice (compare the third and fourth rows from the bottom). These results indicate that HS does not sensitize mice to TNF- $\alpha$-induced liver injury and apoptosis.

\section{Effects of HS on Caspase Activities}

To assess whether caspases are involved in the apoptotic process induced by GalN plus TNF- $\alpha$ in mice pretreated with or without HS, the activities of ALT, caspase-8-like and -3-like proteases were determined (Figure 2). The activities of caspase-8like and -3-like proteases increased at 4 and $6 \mathrm{~h}$ after administration of GalN plus TNF- $\alpha$. In mice challenged with prior HS for 7 days, the activities of caspase-8-like and -3-like proteases were promoted to a greater extent at $4 \mathrm{~h}$ after GalN plus TNF- $\alpha$ than in mice without prior HS. No significant difference was found at $6 \mathrm{~h}$ after the administration between HS-pretreated and -nontreated mice. More severe liver damage was observed in mice pretreated with repeated HS at $6 \mathrm{~h}$ after GalN plus TNF- $\alpha$ adminis- tration compared with mice without prior HS (Figure 2a). This finding may indicate that the decreases in caspase-8-like and -3-like activities at $6 \mathrm{~h}$ following GalN plus TNF- $\alpha$ treatment in mice pretreated with repeated HS are probably due to the more severe liver damage. We found that the activity of caspase-8-like protease was already upregulated in mice pretreated with HS before the administration of GalN plus TNF- $\alpha$, indicating that caspase8-like protease was constitutively activated in repeated HS-challenged mice.

\section{HS Treatment Induces HSP70 and Caspase-8 Activity}

To investigate whether the HS conditions used in the present study led to HSP70 induction and caspase activation, mice were killed $12 \mathrm{~h}$ after the final HS treatment. The livers were removed and homogenized, and the homogenates were subjected to Western blot analysis for HSP70, procaspase-8, and p20, which is the cleaved product of procaspase-8 (Figure 3a). The level of HSP70 expression in the liver rose with the number of HS treatments. Similarly, cleavage of procaspase-8 in the liver was
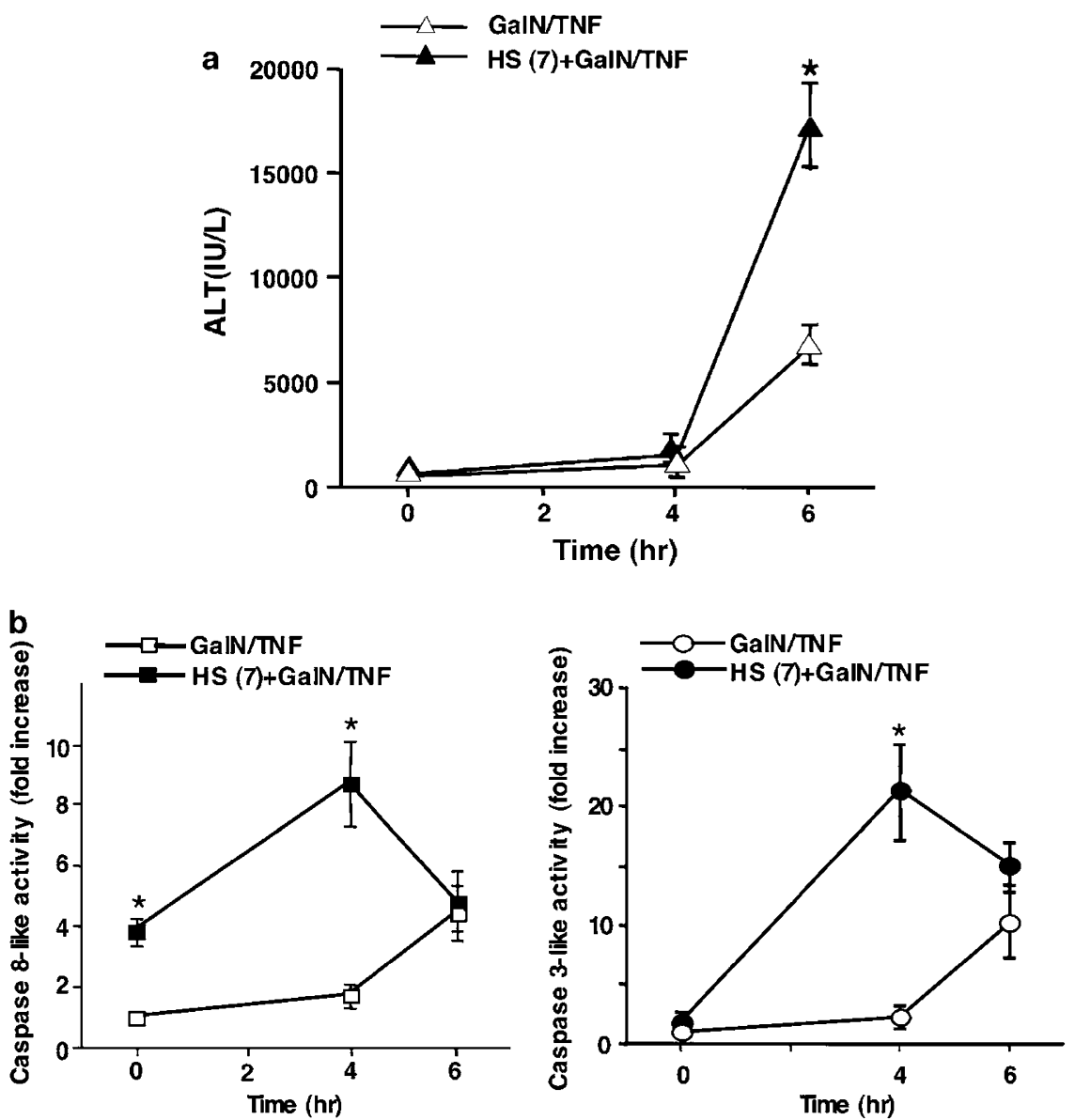

Figure 2 HS promotes activation of liver caspases. Assessment of (a) ALT and (b) caspase-8-like and caspase-3-like activity in the liver collected at the indicated time points after administration with GalN/TNF- $\alpha$ in the presence or absence of HS pretreatment for 7 days. Data are expressed as the mean \pm s.e. for $3-6$ mice. ${ }^{*} P<0.05$ compared to mice treated with GalN/TNF- $\alpha$. 

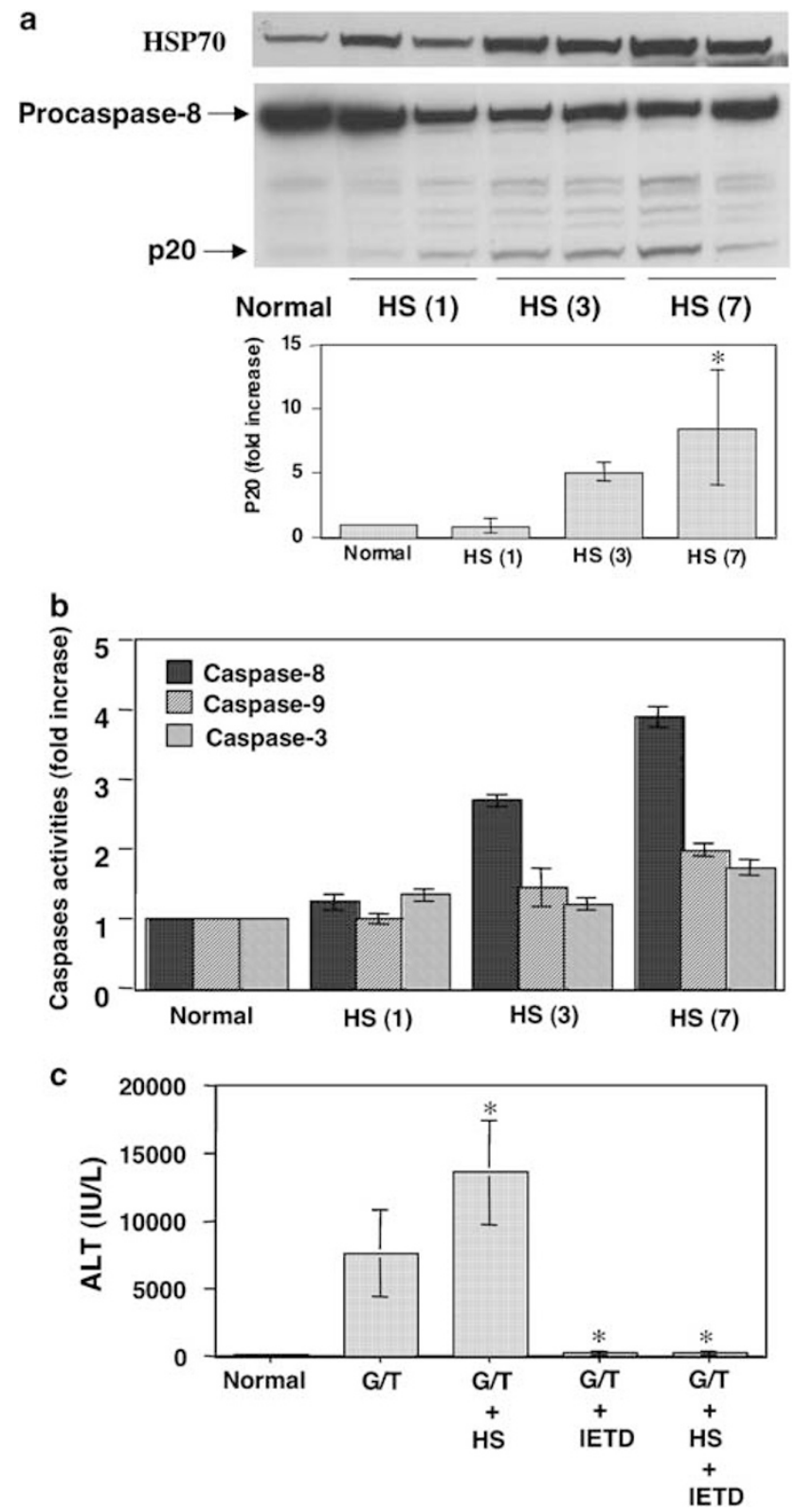

Figure 3 Treatment with HS for 7 days increases HSP70 and caspase-8 activity. (a) Induction of HSP70 and cleavage of procaspase-8 in the liver of mice after whole-body HS. Total liver proteins $(50 \mu \mathrm{g})$ from the mice were subjected to SDS-PAGE, and were probed with anti-HSP70 or antiprocaspase-8. The results shown are representative of at least three independent experiments. Analysis of the processed form of the caspase-8 (p20) band was performed on a Macintosh computer using the public domain $\mathrm{NIH}$ Image program. Data are expressed as the mean \pm s.e. for 3-4 mice. ${ }^{*} P<0.05$ compared to mice treated with HS for 1 day. (b) Effects of HS on caspase-8-like, -9-like, and -3-like activity in the mouse liver. For measurement of caspase-8-like, -9-like, and -3like protease activities, the synthetic fluorogenic substrates, AcIETD-AMC, Ac-LEHD-AMC and Ac-DEVD-AMC were used, respectively. Data are expressed as the mean \pm s.e. for three to four mice. (c) Effects of the caspase-8 inhibitor Z-IETD-FMK on the hepatotoxicity of mice induced by GalN/TNF- $\alpha$ with or without prior HS treatment. Blood was collected $6 \mathrm{~h}$ after GalN/ TNF- $\alpha$ administration, and serum alanine aminotransferase activities were determined as indices of hepatotoxicity. Data are expressed as the mean \pm s.e. for three to four mice. ${ }^{*} P<0.05$ compared to mice treated with GalN/TNF- $\alpha$.

induced by HS treatment. The cleavage was maximal in the liver treated with HS for 7 days. Consistent with the Western blot analysis, the activity of caspase-8 (-like) protease drastically increased in the livers of mice treated with HS for 7 days (Figure 3b). However, only a slight increase in the activity of caspase-9 and -3 (-like) proteases occurred in the livers of mice treated with HS. To investigate the role of caspase- 8 in the liver injury induced by GalN/TNF- $\alpha$ and HS for 7 days, we evaluated the effect of caspase-8 inhibitor Z-IETD-FMK on the hepatotoxicity (Figure 3c). The caspase-8 inhibitor effectively protected mice from the hepatotoxicity induced by GalN/TNF- $\alpha$ and HS for 7 days and also from that induced by GalN/TNF$\alpha$ alone. These findings show that TNF- $\alpha$-induced liver injury associated with HS is caspase-8dependent.

\section{Effects of HS on the Expressions of FLIP and Akt in the Liver}

The above results indicate that severe HS may promote TNF- $\alpha$-induced liver injury through activation of caspase-8. Hence, we determined the expressions of FLIP, a physiological inhibitor of caspase-8, and Akt, which is known to regulate FLIP. ${ }^{20}$ As shown in Figure 4a, the level of FLIP ${ }_{S}$ was decreased by single HS treatment, and decreased further by multiple HS treatment, although HS had no effect on the level of $\mathrm{FLIP}_{\mathrm{L}}$. Akt phosphorylation occurred in the normal liver, indicating that Akt is constitutively activated in the liver, and HS decreased the levels of phosphorylated Akt as well as those of Akt. In addition, severe HS decreased the

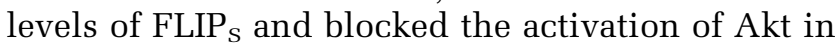
the liver of mice treated with GalN plus TNF- $\alpha$ (Figure 4b). The release of cytochrome $c$ to the cytosol was little observed when sequences of HS were given to mice. In contrast, cytochrome $C$ content in the cytosolic fraction of the liver dramatically increased with a reciprocal decrease in the membrane fraction of liver in either mice pretreated with or without repeated HS after GalN/ TNF- $\alpha$ administration, indicating that hepatocyte apoptosis is induced via mitochondria-dependent pathway in the livers of mice treated with repeated HS and GalN/TNF- $\alpha$ comparably in those of mice treated with GalN/TNF- $\alpha$ alone.

\section{Discussion}

This study demonstrated that HS promoted or prevented TNF- $\alpha$-mediated liver cell death depending on the duration of exposure. We found that repeated exposure to HS led to significant enhancement of TNF- $\alpha$-induced hepatocyte apoptosis, whereas exposure to single HS protected against such apoptosis. The death-promoting effect of HS observed here is at variance with the commonly 


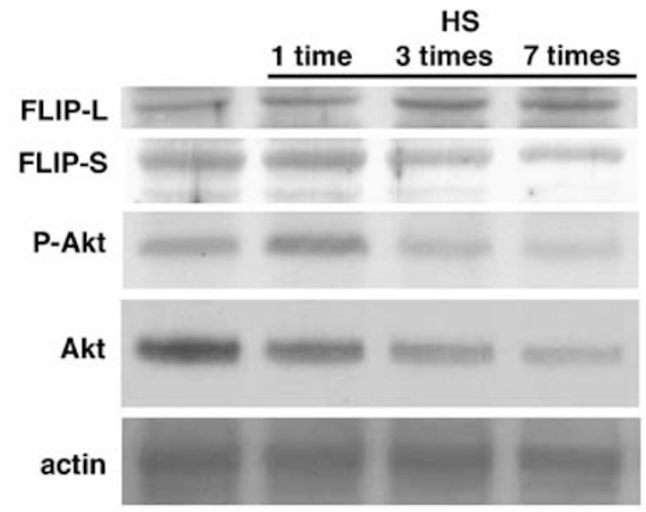

Cyt.c membrane cytosol

b

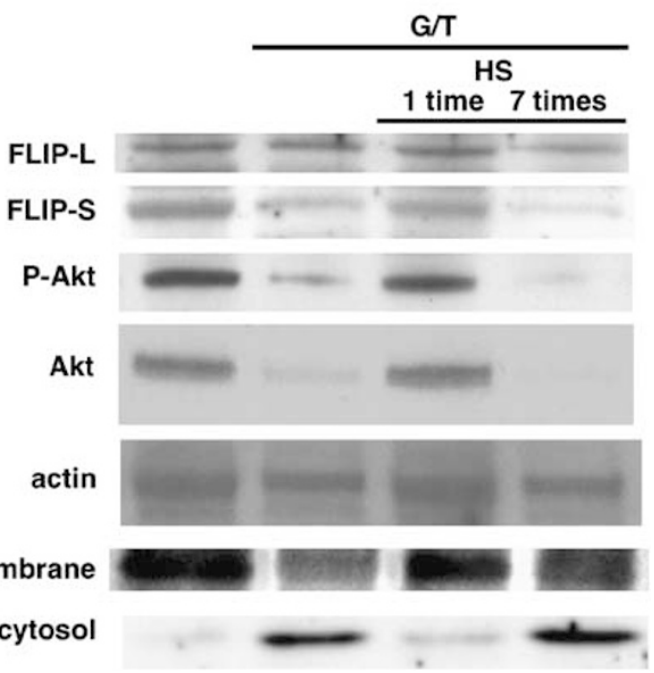

Figure 4 HS effectively blocked FLIP $_{S}$ and Akt phosphorylation in the liver of untreated mice (a) and mice treated with GalN/ TNF- $\alpha$ (b). Total liver proteins $(50 \mu \mathrm{g})$ from the mice were subjected to SDS-PAGE, and were probed with anti-FLIP, , - FLIP $_{\mathrm{L}}$, anti-Akt, antiphosphorylated-Akt, or anticytochrome $c$. The results shown are representative of at least three independent experiments.

described protective effect of HSP70 induced by HS. Molle et $a l^{10}$ have recently demonstrated that HS treatment of mice leads to a strong induction of HSP70 in several organs and confers significant protection against lethality induced by TNF- $\alpha$. In addition, they have shown that mice deficient in the inducible hsp70.1 gene were no longer protected by HS treatment, indicating that HSP70 is at least necessary for the conferred protection induced by whole-body HS of mice. Therefore, it was surprising to observe that HS, with subsequent HSP70 induction, promoted TNF- $\alpha$-induced hepatocyte apoptosis rather than protecting against such hepatotoxicity. However, this discrepancy could be explained by the differing degrees of HS exposure. We found that single HS suppressed the hepatotoxicity, whereas repeated HS (HS performed for 7 days) promoted it (Table 1). A future experiment should be performed in $\mathrm{HSP}^{-/-}$mouse to address whether HSP70 induction per se is responsible for exacerbating the liver injury after repeated HS as well as attenuates the injury induced by a single exposure to HS.

Consistent with our results, DeMeester et $a l^{20}$ have demonstrated that induction of HS in cells primed by inflammation can precipitate cell death by apoptosis, whereas a prior heat shock stress can protect cell against inflammatory stress both in vitro and in vivo. They called this seemingly paradoxical ability of HS to induce cytoprotection and cytotoxicity the 'heat shock paradox'. However, Molle et al ${ }^{10}$ demonstrated that HS treated for 10 consecutive days did not lead to downregulation of HSP 70 expression, and still conferred protection against TNF lethality. It is difficult to precisely explain this discrepancy with our findings. Although they used TNF- $\alpha$ alone and did not show liver injury, we administrated to mice TNF- $\alpha$ plus GalN, which is well known to increase the susceptibility of mice especially to liver damage and hepatocyte apoptosis in response to TNF- $\alpha .{ }^{21}$ A sequence of stressful stimuli can produce unexpected responses at the cellular level, and may therefore result in different phenotypic responses in different organs or cells. For example, since caspases are the main executers of apoptosis, any sensitizing stimulus may affect the degree of activation of one or more caspases. In the present study, the activities of caspases-8 and -3 increased to a greater extent in repeated HS-challenged mice than in HS-nonchallenged mice. Moreover, the observation that caspase-8 was already cleaved and activated in the absence of apoptosis in heat-shocked samples of livers indicates that caspase-8 cleavage can be the cause of the acceleration, rather than its consequence.

This hypothesis is supported by the fact that HS specifically activated caspase-8, but not caspase-3 or -9. Moreover, administration of caspase-8 inhibitor prevented GalN/TNF- $\alpha$-induced liver injuries both in HS-treated and nontreated mice, indicating that caspase-8 is the initial caspase in HS-mediated acceleration of hepatocytes in response to TNF$\alpha$-induced apoptosis, and suggesting that the effect of HS on hepatocyte apoptosis was upstream of caspase-8 activation. Many studies have demonstrated that caspase-8 expression acts as a key determinant of sensitivity for apoptosis induced by death-inducing ligands or cytotoxic drugs. ${ }^{22-24} \mathrm{IFN}-\gamma$ sensitizes tumor cells resistant to apoptosis by upregulating caspase-8 expression through the signal transducer and activator of transcription-1 (STAT1) pathway. ${ }^{25}$ The LF15-0195 immunosuppressive agent sensitizes Jurkat $\mathrm{T}$ cells to apoptosis induced by an anti-CD95 antibody and by the TNFrelated apoptosis-inducing ligand by facilitating caspase-8 activation at the death-inducing signaling complex (DISC). ${ }^{26}$

To further investigate the mechanism of HSmediated caspase-8 activation, we examined the involvement of the DISC protein in this process, 


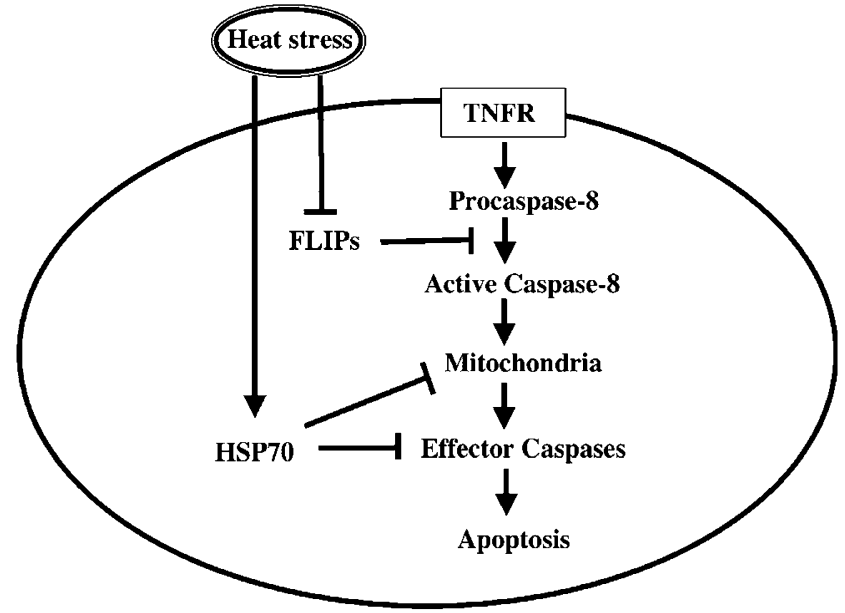

Figure 5 Proposed proapoptotic role of HS in TNF- $\alpha$-induced hepatocyte apoptosis. Repeated HS simultaneously induces HSP70 and suppresses FLIP $_{S}$, resulting in the activation of caspase- 8 in cells. These changes in the cells lead to enhancement of TNF- $\alpha$-induced hepatocyte apoptosis.

since FLIP has been characterized as an endogenous caspase-8 inhibitor. ${ }^{27}$ FLIP has been shown to correlate with resistance of cells against Fasmediated apoptosis,$^{28}$ and downregulation of FLIP sensitizes a variety of endothelial cells and tumor cells to death receptor-mediated apoptosis. ${ }^{29-32}$ Tran et $a l^{18}$ have demonstrated that HS downregulates FLIP and sensitizes Jurkat cells to Fas-mediated apoptosis, which is consistent with our results. In most of the studies, however, FLIP-related sensitization to apoptosis has been shown in vitro. We here showed in vivo that repeated HS, but not single HS, downregulated FLIP and promoted TNF- $\alpha$-induced liver cell death. FLIP is expressed in two isoforms, FLIP $_{L}$ and FLIP , both of which can inhibit caspase8 activation. ${ }^{33}$ FLIP $_{\mathrm{L}}$ consists of two N-terminal death effector domains and a short C-terminal caspase homology domain devoid of enzymatic activity, whereas FLIP $_{S}$ is only composed of the N-terminal death effector domains and a short C-terminal stretch of amino acids not found in FLIP $_{\mathrm{L}}$. Both FLIP $\mathrm{F}_{\mathrm{L}}$ and FLIP $\mathrm{S}$ can be recruited to the DISC but they function differently. ${ }^{34}$ It has been reported that each of the FLIP proteins can be involved in susceptibility to death receptormediated apoptosis. ${ }^{18,24,35}$ In the present study, FLIP $_{S}$ was downregulated by moderate HS, and further downregulated by repeated HS although FLIP $_{\mathrm{L}}$ was little changed (Figure 4a). This correlated with caspase-8 activation and apoptosis. These data suggest that downregulation of FLIP $_{s}$, rather than FLIP $_{\mathrm{L}}$, promotes TNF- $\alpha$-induced hepatocyte apoptosis in repeated HS-treated mice.

The levels of FLIP S expression were associated with those of Akt activity, which is well known to regulate FLIP expression, ${ }^{36,37}$ but those of FLIP $_{\mathrm{L}}$ were not. It has been reported that FLIP $_{S}$ and FLIP $_{\mathrm{L}}$ are differently regulated, ${ }^{38}$ although the difference in the regulatory mechanisms remains unclear. Our data suggest that Akt activity might regulate only FLIP $_{S}$, but not FLIP $_{\mathrm{L}}$ expression, and that this may contribute to the TNF- $\alpha$-resistance in hepatocytes.

In conclusion, we demonstrated that repeated HS enhanced TNF- $\alpha$-induced hepatotoxicity in GalNsensitized mice through the activation of caspase-8 and the suppression of FLIP $_{S}$ expression (Figure 5), in contradiction to the well established finding that prior heat shock stress protects cells via induction of HSPs. We believe that these results will help to provide an understanding of the mechanism of balance between cell death and viability, and provide important clues regarding the differential stress response interactions between single and sequential stimuli.

\section{Acknowledgements}

This work was supported in part by a grant-in-aid from the Ministry of Education, Science, Sports, and Culture of Japan (Grant 15590636) and by the Research Group of Intractable Liver Diseases sponsored by the Ministry of Health and Welfare of Japan.

\section{References}

1 Lindquist S, Craig EA. The heat-shock proteins. Annu Rev Genet 1988;22:631-677.

2 Benjamin IJ, McMillan DR. Stress (heat shock) proteins: molecular chaperones in cardiovascular biology and disease. Circ Res 1998;83:117-132.

3 Beere HM, Wolf BB, Cain K, et al. Heat-shock protein 70 inhibits apoptosis by preventing recruitment of procaspase-9 to the Apaf-1 apoptosome. Nat Cell Biol 2000;2:469-475.

4 Saleh A, Srinivasula SM, Balkir L, et al. Negative regulation of the Apaf-1 apoptosome by Hsp70. Nat Cell Biol 2000;2:476-483.

5 Li F, Mao HP, Ruchalski KL, et al. Heat stress prevents mitochondrial injury in ATP-depleted renal epithelial cells. Am J Physiol Cell Physiol 2002;283: C917-C926.

6 Gabai VL, Meriin AB, Mosser DD, et al. Hsp70 prevents activation of stress kinases. A novel pathway of cellular thermotolerance. J Biol Chem 1997;272: 18033-18037.

7 Mosser DD, Caron AW, Bourget L, et al. Role of the human heat shock protein hsp70 in protection against stress-induced apoptosis. Mol Cell Biol 1997; 17:5317-5327.

8 Meriin AB, Yaglom JA, Gabai VL, et al. Proteindamaging stresses activate c-Jun N-terminal kinase via inhibition of its dephosphorylation: a novel pathway controlled by HSP72. Mol Cell Biol 1999;19: 2547-2555.

9 Gabai VL, Mabuchi K, Mosser DD, et al. Hsp72 and stress kinase c-jun N-terminal kinase regulate the biddependent pathway in tumor necrosis factor-induced apoptosis. Mol Cell Biol 2002;22:3415-3424. 
10 Van Molle W, Wielockx B, Mahieu T, et al. HSP70 protects against TNF-induced lethal inflammatory shock. Immunity 2002;16:685-695.

11 Hotchkiss R, Nunnally I, Lindquist S, et al. Hyperthermia protects mice against the lethal effects of endotoxin. Am J Physiol 1993;265(6 Part 2):R1447-R1457.

12 Mikami K, Otaka M, Goto $\mathrm{T}$, et al. Induction of a $72-\mathrm{kDa}$ heat shock protein and protection against lipopolysaccharide-induced liver injury in cirrhotic rats. J Gastroenterol Hepatol 2004;19:884-890.

13 Nagaki M, Naiki T, Brenner DA, et al. Tumor necrosis factor alpha prevents tumor necrosis factor receptormediated mouse hepatocyte apoptosis, but not fasmediated apoptosis: role of nuclear factor-kappaB. Hepatology 2000;32:1272-1279.

14 Liossis SN, Ding XZ, Kiang JG, et al. Overexpression of the heat shock protein 70 enhances the TCR/CD3- and Fas/Apo-1/CD95-mediated apoptotic cell death in Jurkat T cells. J Immunol 1997;158:5668-5675.

15 Schett G, Steiner CW, Xu Q, et al. TNFalpha mediates susceptibility to heat-induced apoptosis by protein phosphatase-mediated inhibition of the HSF1/hsp70 stress response. Cell Death Differ 2003;10:1126-1136.

16 Chant ID, Rose PE, Morris AG. Susceptibility of AML cells to in vitro apoptosis correlates with heat shock protein 70 (hsp 70) expression. Br J Haematol 1996;93: 898-902.

17 DeMeester SL, Buchman TG, Qiu Y, et al. Pyrrolidine dithiocarbamate activates the heat shock response and thereby induces apoptosis in primed endothelial cells. Shock 1998;10:1-6.

18 Tran SE, Meinander A, Holmstrom TH, et al. Heat stress downregulates FLIP and sensitizes cells to Fas receptor-mediated apoptosis. Cell Death Differ 2003; 10:1137-1147.

19 Ran R, Lu A, Zhang L, et al. Hsp70 promotes TNFmediated apoptosis by binding IKK gamma and impairing NF-kappa B survival signaling. Genes Dev 2004;18:1466-1481.

20 DeMeester SL, Buchman TG, Cobb JP. The heat shock paradox: does NF-kappaB determine cell fate? FASEB J 2001;15:270-274.

21 Leist M, Gantner F, Kunstle G, et al. The 55-kD tumor necrosis factor receptor and CD95 independently signal murine hepatocyte apoptosis and subsequent liver failure. Mol Med 1996;2:109-124.

22 Fulda S, Kufer MU, Meyer E, et al. Sensitization for death receptor- or drug-induced apoptosis by reexpression of caspase-8 through demethylation or gene transfer. Oncogene 2001;20:5865-5877.

23 Kim PK, Mahidhara R, Seol DW. The role of caspase-8 in resistance to cancer chemotherapy. Drug Resist Updat 2001;4:293-296.

24 Hougardy BM, van der Zee AG, van den Heuvel FA, et al. Sensitivity to Fas-mediated apoptosis in high-risk HPV-positive human cervical cancer cells: relationship with Fas, caspase-8, and Bid. Gynecol Oncol 2005;97:353-364.

25 Fulda S, Debatin KM. IFNgamma sensitizes for apoptosis by upregulating caspase-8 expression through the Stat1 pathway. Oncogene 2002;21: 2295-2308.

26 Ducoroy P, Micheau O, Perruche S, et al. LF 15-0195 immunosuppressive agent enhances activationinduced T-cell death by facilitating caspase-8 and caspase-10 activation at the DISC level. Blood 2003; 101:194-201.

27 Irmler M, Thome M, Hahne M, et al. Inhibition of death receptor signals by cellular FLIP. Nature 1997;388:190-195.

28 Schmitz I, Weyd H, Krueger A, et al. Resistance of short term activated T cells to CD95-mediated apoptosis correlates with de novo protein synthesis of c-FLIPshort. J Immunol 2004;172:2194-2200.

29 Kim Y, Suh N, Sporn M, et al. An inducible pathway for degradation of FLIP protein sensitizes tumor cells to TRAIL-induced apoptosis. J Biol Chem 2002;277: 22320-22329.

30 Erwert RD, Winn RK, Harlan JM, et al. Shiga-like toxin inhibition of FLICE-like inhibitory protein expression sensitizes endothelial cells to bacterial lipopolysaccharide-induced apoptosis. J Biol Chem 2002;277: 40567-40574.

31 Perez D, White E. E1A sensitizes cells to tumor necrosis factor alpha by downregulating c-FLIP S. J Virol 2003;77:2651-2662.

32 Watanabe K, Okamoto K, Yonehara S. Sensitization of osteosarcoma cells to death receptor-mediated apoptosis by HDAC inhibitors through downregulation of cellular FLIP. Cell Death Differ 2005;12:10-18.

33 Krueger A, Schmitz I, Baumann S, et al. Cellular FLICE-inhibitory protein splice variants inhibit different steps of caspase-8 activation at the CD95 deathinducing signaling complex. J Biol Chem 2001;276: 20633-20640.

34 Wajant $H$. Targeting the FLICE Inhibitory Protein (FLIP) in cancer therapy. Mol Interv 2003;3:124-127.

35 Kinoshita H, Yoshikawa H, Shiiki K, et al. Cisplatin (CDDP) sensitizes human osteosarcoma cell to Fas/ CD95-mediated apoptosis by down-regulating FLIP-L expression. Int J Cancer 2000;88:986-991.

36 Nam SY, Jung GA, Hur GC, et al. Upregulation of FLIP(S) by Akt, a possible inhibition mechanism of TRAIL-induced apoptosis in human gastric cancers. Cancer Sci 2003;94:1066-1073.

37 Kang YC, Kim KM, Lee KS, et al. Serum bioactive lysophospholipids prevent TRAIL-induced apoptosis via PI3K/Akt-dependent cFLIP expression and Bad phosphorylation. Cell Death Differ 2004;11:1287-1298.

38 Bin L, Li X, Xu LG, et al. The short splice form of Casper/c-FLIP is a major cellular inhibitor of TRAILinduced apoptosis. FEBS Lett 2002;510:37-40. 